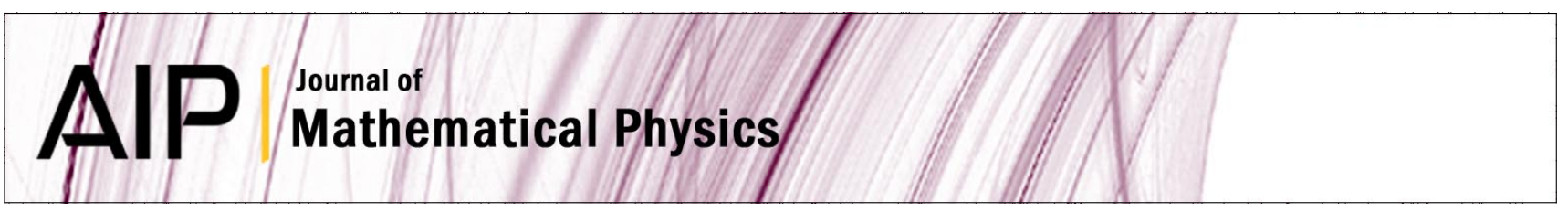

\title{
Sparse one-dimensional discrete Dirac operators II: Spectral properties
}

S. L. Carvalho, C. R. de Oliveira, and R. A. Prado

Citation: J. Math. Phys. 52, 073501 (2011); doi: 10.1063/1.3600536

View online: http://dx.doi.org/10.1063/1.3600536

View Table of Contents: http://jmp.aip.org/resource/1/JMAPAQ/v52/i7

Published by the AIP Publishing LLC.

\section{Additional information on J. Math. Phys.}

Journal Homepage: http://jmp.aip.org/

Journal Information: http://jmp.aip.org/about/about_the_journal

Top downloads: http://jmp.aip.org/features/most_downloaded

Information for Authors: http://jmp.aip.org/authors

\section{ADVERTISEMENT}

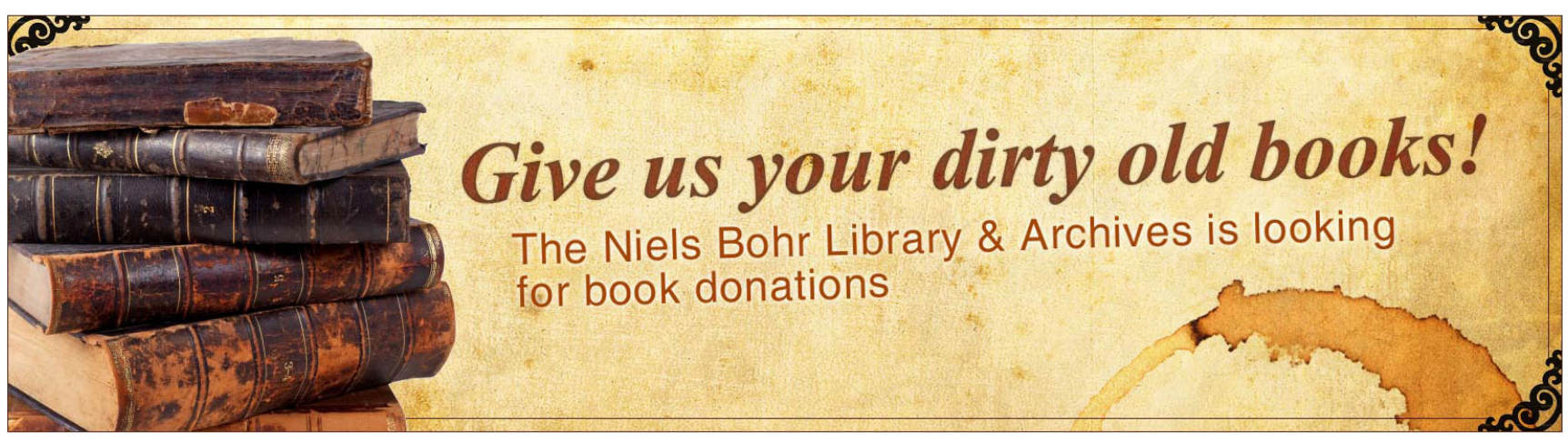




\title{
Sparse one-dimensional discrete Dirac operators II: Spectral properties
}

\author{
S. L. Carvalho, ${ }^{1, a)}$ C. R. de Oliveira, ${ }^{1, b)}$ and R. A. Prado ${ }^{2, c)}$ \\ ${ }^{1}$ Departamento de Matemática - UFSCar, São Carlos, SP 13560-970, Brazil \\ ${ }^{2}$ Departamento de Matemática, Estatística e Computação - UNESP, Presidente Prudente, \\ SP 19060-900, Brazil
}

(Received 14 March 2011; accepted 24 May 2011; published online 5 July 2011)

\begin{abstract}
We study spectral properties of some discrete Dirac operators with nonzero potential only at some sparse and suitably randomly distributed positions. As observed in the corresponding Schrödinger operators, we determine the Hausdorff dimension of its spectral measure and identify a sharp spectral transition from point to singular continuous. () 2011 American Institute of Physics. [doi:10.1063/1.3600536]
\end{abstract}

\section{INTRODUCTION}

Sparse potentials have played a special role in the context of discrete (also called "tightbinding") Schrödinger operators, particularly due to the possibility of rather detailed spectral and dynamical analysis, which makes such investigations especially interesting (see, for instance, Refs. 1, 4, 12, 16, 20, 28, and 31). Pearson ${ }^{21}$ was the first to recognize the utility of this kind of potential on the construction of Schrödinger operators with singular continuous spectrum (see Chapter 13 in Ref. 6 for dynamical interpretations of different spectral types). There are many works that deal with sparse potentials, related to distinct sparseness conditions and "barriers" with distinct growing rate (see the above mentioned references and Ref. 18 for a brief discussion and a collection of results).

In the works, ${ }^{7,8}$ two of the present authors have proposed a discrete version of the unidimensional Dirac operator, now defined on $l^{2}\left(\mathbb{Z}, \mathbb{C}^{2}\right)$, which can be considered a relativistic version of the usual discrete Schrödinger operator (with Planck's constant $\hbar \equiv 1$ ),

$$
\left(H_{S} \psi\right)_{n}=-\frac{1}{2 m}(\Delta \psi)_{n}+V_{n} \psi_{n}=\frac{1}{2 m}\left(\psi_{n+1}+\psi_{n-1}-2 \psi_{n}\right)+V_{n} \psi_{n},
$$

where $\left(V_{n}\right)$ is the potential given by a real sequence and $m>0$ denotes the particle mass. Explicitly, the action of the discrete Dirac operator is given by

$$
\left(H_{D}\right)(m, c)=H_{0}(m, c)+V \mathrm{I}_{2}=c \mathcal{B}+m c^{2} \sigma_{3}+V \mathrm{I}_{2},
$$

where $c>0$ represents the speed of light,

$$
\mathcal{B}=\left(\begin{array}{cc}
0 & d^{*} \\
d & 0
\end{array}\right),
$$

$\sigma_{3}$ is the Pauli matrix $\left(\begin{array}{cc}1 & 0 \\ 0 & -1\end{array}\right), \mathrm{I}_{2}$ is the $2 \times 2$ identity matrix, and $d$ is the finite difference operator (a discrete version of the first derivative),

$$
(d \psi)_{n}=\psi_{n+1}-\psi_{n},
$$

\footnotetext{
a)Electronic mail: silas@dm.ufscar.br.

b)Electronic mail: oliveira@dm.ufscar.br.

c) Electronic mail: robertoprado@fct.unesp.br
} 
with its adjoint given by $\left(d^{*} \psi\right)_{n}=\psi_{n}-\psi_{n-1}$; thus, $H_{0}(m, c)$ is a bounded self-adjoint operator with the mass parameter taking values $m \geq 0$, an important difference with respect to nonrelativistic models.

The resulting Dirac evolution equation can be written in the compact form,

$$
i \frac{\partial \Psi_{n}}{\partial t}=\left(H_{D}(m, c) \Psi\right)_{n}=\left(\begin{array}{cc}
m c^{2}+V_{n} & c d^{*} \\
c d & -m c^{2}+V_{n}
\end{array}\right) \Psi_{n},
$$

with the "spinorial" wave function $\Psi=\left(\Psi_{n}\right)$, with $\Psi_{n}=\left(\begin{array}{l}\psi_{1, n} \\ \psi_{2, n}\end{array}\right)$, where $\psi_{1, n}$ and $\psi_{2, n}$ are the solutions associated, respectively, with positive and negative energy values (see, for instance, Chapter 3 in Refs. 25 or 29). It is possible to show ${ }^{7,8}$ that the nonrelativistic limit $(c \rightarrow \infty)$ of such Dirac operator is the corresponding Schrödinger operator (1.1), as one would expect.

In a previous work, ${ }^{22}$ some lower bounds of the dynamics generated by $H_{D}(m, c)$ with sparse potentials have been obtained. In the present work, we continue our investigations on the discrete Dirac operator with sparse potentials by presenting a detailed analysis of spectral properties of (1.2) defined in $l^{2}\left(\mathbb{Z}_{+}, \mathbb{C}^{2}\right)$ (here $\mathbb{Z}_{+}$represents the set of all non-negative integers $n \geq 0$ ), satisfying the boundary condition,

$$
\psi_{2,-1} \cos \phi-\psi_{1,0} \sin \phi=0,
$$

with $\phi \in[0, \pi]$, and subject to randomly sparse perturbations composed of infinitely many vertical "barriers" whose distances from consecutive barriers are rapidly growing. These barriers may assume distinct sizes, that grow, diminish, or remain constant. The randomness in the distribution will play a fundamental role in the determination of the exact Hausdorff dimension of the spectral measure. We will consider a set $\left\{a_{j}\right\}_{j \geq 1}$ of a rapidly increasing sequence of natural numbers, so that the potential $V_{n}=0$ if $n \notin\left\{a_{j}\right\}_{j \geq 1}$ and nonzero $V_{a_{j}}$; see ahead for precise statements.

We, nevertheless, emphasize the results obtained by Zlatoš ${ }^{31}$ in the context of Schrödinger operators, who has proved the existence of a transition between pure point and singular continuous spectra for a class of sparse operators such that $\left\{V_{a_{j}}\right\}_{j=1}^{\infty}$ is a constant sequence. Depending on the sparseness and on the intensity of the barriers, it is possible to have pure point or singular continuous spectrum, and with an explicit determination of the Hausdorff dimension of the spectral measure.

A similar deterministic model was proposed in Ref. 20, but with off-diagonal perturbations. Carvalho et al. ${ }^{1}$ extended this operator to the strip $\Lambda:=\mathbb{Z}_{+} \times\{0,1, \ldots, L-1\}$ of width $L$ in the $\mathbb{Z}_{+}^{2}$ plane, obtaining the Hausdorff dimension associated with its spectral measure with arbitrary precision. In Ref. 2, the same authors have dealt with a modification of the (unidimensional) Schrödinger model proposed in Ref. 20, in which they have considered some randomness in the position of the barriers, and this has allowed the determination of the exact Hausdorff dimension of the spectral measure.

Hence, by taking into account our previous study, ${ }^{22}$ it is natural to try to extend these spectral results obtained for Schrödinger operators to the Dirac model. In order to achieve our goal, we apply and extend to this new situation the main techniques discussed in Refs. 1 and 2. This work is to be considered a natural continuation of Ref. 22.

Despite some results obtained here are valid for a rather general class of potentials, we deal essentially with the sequence $\left(V_{n}(\omega)\right)$ of barriers given by

$$
V_{n}=\left\{\begin{array}{lll}
v & \text { if } & n=a_{j}^{\omega} \in \mathcal{A} \\
0 & \text { if } & n \notin \mathcal{A}
\end{array}\right.
$$

where $v \neq 0,-\infty<v<\infty$ and $\mathcal{A}=\left(a_{j}^{\omega}\right)_{j \geq 1}$ is a random sequence of natural numbers written in the form $a_{j}^{\omega}=a_{j}+\omega_{j}$ such that $a_{j}$ satisfies

$$
a_{j}-a_{j-1} \geq 2, \quad j=2,3, \ldots
$$

and

$$
\lim _{j \rightarrow \infty} \frac{a_{j+1}}{a_{j}}=\beta>1
$$


$\omega=\left(\omega_{1}, \omega_{2}, \ldots\right)$ represents a sequence of independent random variables defined on a probability space $(\Xi, \mathcal{B}, v)$, so that there is some $\eta>0$ with $\omega_{j}$ uniformly distributed over the finite set $\left\{0,1,2, \ldots,\left[j^{\eta}\right]\right\}$, for all $j$ (here, $[x]$ denotes the integer part of $x \in \mathbb{R}$ ).

Condition (1.6) makes each barriers placed at a unique point, with $\beta$ the "sparseness parameter." In order to simplify our analysis, we follow ${ }^{20}$ and restrict the separation between barriers by the identity

$$
a_{j}-a_{j-1}=\beta^{j}, \quad j=2,3, \ldots
$$

with $a_{1}+1=\beta \geq 2$ an integer (we might have considered any real number $\beta>1$ and rewrite (1.7) as $a_{j}-a_{j-1}=\left[\beta^{j}\right], j=2,3, \ldots$ ). Summing up, the distance between (average) consecutive nonzero potential positions grows exponentially with an additional power-law randomness; thus, there is no superpositions among the barriers, for any $\eta>0$ (this is an improvement with respect to Zlatoš's work that has considered $\eta=1$ ).

Definition 1.1: Denote by $H_{v, \phi}(m, c)$, the Dirac operator (1.2) acting on $l^{2}\left(\mathbb{Z}_{+}, \mathbb{C}^{2}\right)$, with potential $\left(V_{n}(\omega)\right)_{n \geq 0}$ satisfying (1.5) and (1.7), and the $\phi$-boundary condition (1.4) at $n=-1$. This will be the main object of study here.

Besides the verification of a huge amount of technical details and suitable adaptations, the main difficulties we have found in such extension to the (Dirac) relativistic framework were as follows:

- The nonlinearities in the Dirac transfer matrices entries (as functions of energy and potential values) when compared with the Schrödinger case; see Eq. (3.2).

- In contrast to the block-Jacobi matrices in Ref. 1, the spectral matrix $\Omega$ in the Dirac case (see Proposition 2.3) reflects the impossibility of decoupling the upper and lower spinor components.

- Finding explicit expressions for the Green's function; see Eq. (2.5) (it has involved a trial and error process while handling the exact matrix form and indices!).

- Finding a formula for variation of parameters (Lemma 4.4) was nontrivial and it also involved a trial and error process, and this was fundamental for an appropriate version of the JitomirskayaLast inequalities for Dirac operators (Theorem 4.3).

- Checking the validity of the spectral criteria of Last-Simon (see Proposition 4.5 and Corollary 4.8 for examples of application of these criteria), whose long details are not reported here.

We note that in the process of adaptation of known results to the relativistic setting, we will avoid repeating the arguments and just present references when a proof turns out to be quite similar to the corresponding one for Schrödinger operators (e.g., the spectral criteria of Last-Simon just mentioned).

Remark 1.2: The operator $H_{v, \phi}(m, c)$ with $\phi$-phase boundary condition (1.4) may be treated as a rank-one perturbation of the same Dirac operator with Dirichlet boundary condition,

$$
\psi_{2,-1}=0 .
$$

As a matter of fact, if $H_{v, 0}(m, c)$ represents the operator which satisfies (1.8), we have

$$
H_{v, \phi}(m, c)=H_{v, 0}(m, c)+E_{0} \tan \phi,
$$

where $E_{0}$ is an operator on $l^{2}\left(\mathbb{Z}_{+}, \mathbb{C}^{2}\right)$ given by $\left(E_{0} \Psi\right)_{n}=\left(\begin{array}{c}0 \\ \psi_{2, n} \delta_{n, 0}\end{array}\right), \delta_{n, 0}=1$ if $n=0$ and zero otherwise.

\section{A. Main results}

In this subsection, we state the main results and conclusions of this work. Their proofs will be discussed in Secs. II-IV. Such results can be shortly described as follows: determination of the essential spectrum of $H_{v, \phi}(m, c)$ along with the exact Hausdorff dimension of the spectral measure and proving the existence of a spectral transition. 
What happens with the essential spectrum of $H_{0,0}(m, c)$ (obtained in Proposition (2.13)) when we consider the sparse barriers? This question is settled by the following theorem, whose proof is presented in Subsection II B.

Theorem 1.3: Let $H_{v, \phi}(m, c)$ be the Dirac operator in Definition 1.1 and let

$$
H_{0,0}^{\prime}(m, c)=H_{0,0}(m, c)+v \delta_{0} \mathbf{I}_{2},
$$

where $\left(\delta_{0} \psi\right)_{n}=\delta_{n, 0} \psi_{n}$ for any $\psi \in l^{2}\left(\mathbb{Z}_{+}, \mathbb{C}\right)$. Then, $\sigma_{\mathrm{ess}}\left(H_{v, \phi}(m, c)\right)=\sigma\left(H_{0,0}^{\prime}(m, c)\right)$.

For the Hausdorff dimension, we have

Theorem 1.4: Let $\Omega$ be the spectral matrix measure of $H_{v, \phi}(m, c)$, given by Definition 1.1, and the sparseness parameter $\beta \geq 2$. Then $\Omega$ is absolutely continuous with respect to the spectral measure $\rho$ introduced in (2.7). Given $\varepsilon>0$ and a closed interval of energies

$$
L \subset I=\left[-\sqrt{m^{2} c^{4}+4 c^{2}},-m c^{2}\right] \cup\left[m c^{2}, \sqrt{m^{2} c^{4}+4 c^{2}}\right],
$$

for almost every $\phi \in[0, \pi]$ and almost every $\omega \in \Xi$, the spectral measure $\rho$ restrict to $L$ has Hausdorff dimension

$$
\alpha_{\rho}(E)=\max \left\{0,1-\frac{\ln r}{\ln \beta}\right\}
$$

with $r=r(v, E)$ given by (3.16).

With respect to the existence of spectral transitions, now we state a result parallel to Theorem 2.4 in Ref. 2.

Theorem 1.5: Write

$$
I_{1} \equiv\left\{E \in \sigma\left(H_{0,0}(m, c)\right) \backslash A: r<\beta\right\}
$$

with $\beta \in \mathbb{N}, \beta \geq 2$ and $A$ as in (4.13). Then, for $v$-almost every $\omega \in \Xi$, there exists a set $A_{1}$ of Lebesgue measure zero such that:

(a) the spectrum of $H_{v, \phi}(m, c)$ restricted to the set $I_{1} \backslash A_{1}$ is purely singular continuous;

(b) the spectrum of $H_{v, \phi}(m, c)$ is pure point when restricted to $\sigma\left(H_{0,0}(m, c)\right) \backslash I_{1}$ for almost every $\phi \in[0, \pi]$.

Theorem 1.5 shows that there exists a sharp transition between singular continuous and pure point spectrum. Note from (1.11) that the condition for the Hausdorff dimension to be positive is the same for the existence of singular continuous spectrum, i.e., $\beta>r$. In fact, the set of energies for which the Hausdorff dimension is zero coincides with the set where the pure point spectrum is supported. This result, due to Theorem 1.5 is, nevertheless, far from trivial. Note also that there is no absolutely continuous spectrum for this class of sparse potentials.

The proofs of Theorems 1.4 and 1.5 are both presented in Subsection IV B. Despite some obvious differences due to the intrinsic nature of the Schrödinger and Dirac tight-binding models, if we compare the results just stated with those obtained in Refs. 1,2, and 31 for sparse potentials satisfying relations (1.5)-(1.7), we have got quite similar statements; this does not seem to be clear from the actions of the corresponding operators and the different forms of their transfer matrices. In any event, recall that only in the relativistic case it is meaningful to consider $m=0$.

\section{THE SPECTRUM OF $H_{v, \phi}(m, c)$}

\section{A. Green function and the spectral matrix}

It is well known that the spectral properties of any other self-adjoint operator are directly related to the behavior of the Green function $\mathcal{G}(z)$, for $z=E+i \varepsilon$, in the limit $\varepsilon \downarrow 0$. 
In the case of the discrete Dirac operator, the expression of the Green function $\mathcal{G}(z)$ we introduce here is nothing but the $2 \times 2$ matrix $\mathcal{G}(0,0 ; z)$, whose elements are given by

$$
\mathcal{G}_{m, n}(0,0 ; z)=\left\langle e_{m} \otimes \delta_{0},\left(H_{v, 0}-z \mathrm{I}_{2}\right)^{-1} e_{n} \otimes \delta_{0}\right\rangle,
$$

$m, n=1,2$, where the sequence $\left\{\delta_{n}\right\}_{n \geq 0}$ represents the canonical basis for $l^{2}\left(\mathbb{Z}_{+}, \mathbb{C}\right)$, while $e_{1}$ $=\left(\begin{array}{l}1 \\ 0\end{array}\right), e_{2}=\left(\begin{array}{l}0 \\ 1\end{array}\right)$ is the canonical basis for $\mathbb{C}^{2}$; thus, the sequence $\left\{e_{1} \otimes \delta_{n}, e_{2} \otimes \delta_{n}\right\}_{n \geq 0}$ constitutes a basis for $l^{2}\left(\mathbb{Z}_{+}, \mathbb{C}^{2}\right)$, a linear space with scalar product denoted by $\langle\cdot, \cdot\rangle$.

Remark 2.1: The results presented in this subsection refer to the operator $H_{v, 0}(m, c)$, with Dirichlet boundary condition (1.8). See Remark 2.7 for a discussion of the results regarding the operator $H_{v, \phi}(m, c)$.

Explicitly, $\mathcal{G}(z)$ is given by

$$
\mathcal{G}(z)=\frac{1}{c}\left(\begin{array}{ll}
u_{1,0}^{D}(z) \chi_{1,0}(z) & u_{1,0}^{D}(z) \chi_{2,0}(z) \\
u_{1,0}^{D}(z) \chi_{2,0}(z) & u_{2,0}^{D}(z) \chi_{2,0}(z)
\end{array}\right)
$$

where $\chi_{n}(z)=\left(\begin{array}{l}\chi_{1, n}(z) \\ \chi_{2, n}(z)\end{array}\right)=-u_{n}^{N}(z)+m(z) u_{n}^{D}(z)$ is a $l^{2}\left(\mathbb{Z}_{+}, \mathbb{C}^{2}\right)$ is the solution to the Dirac equation

$$
H_{v, 0} \Psi=z \Psi
$$

for some fixed $z$, the set of all solutions to (2.3) is a linear space of dimension 2 (the Dirac equation (2.3) is in fact a system of two differential equations of first order), whose appropriate basis is given by the functions $u^{D}(z)$ and $u^{N}(z)$ satisfying the initial conditions,

$$
\begin{array}{ll}
u_{2,-1}^{D}=0, & u_{1,0}^{D}=1, \\
u_{2,-1}^{N}=1, & u_{1,0}^{N}=0,
\end{array}
$$

which correspond to Dirichlet and Neumann boundary conditions, respectively; finally, $m(z)$ is the well-known Weyl-Titchmarsh function. ${ }^{3}$

Equation (2.1) is in fact a special case derived from the general formula

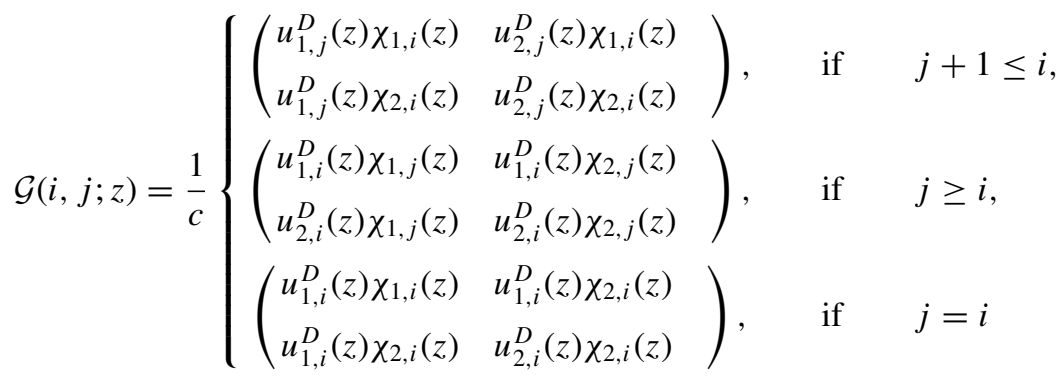

(note that the matricial function defined above is continuous at $j=i$ ).

Remark 2.2: The matrix $\mathcal{G}(i, j ; z)$ is nothing but the integral kernel of the resolvent operator $\left(H_{v, 0}-z \mathbf{I}_{2}\right)^{-1}$; therefore, it must satisfy the identity

$$
\left(\sum_{k=0}^{\infty}\left(H_{v, 0}(m, c)\right)_{i k} \mathcal{G}(k, j ; z)\right)_{l m}=\delta_{i j} \delta_{l m},
$$

$i, j \in \mathbb{Z}_{+}, l, m=1,2$. This assertion can be checked by direct substitution.

If $\left\{P_{\Gamma}\right\}$ is a family of spectral projections with respect to the Borel subsets $\Gamma \subset \mathbb{R}$, it follows by the spectral theorem that

$$
\mathcal{G}(z)=\int_{-\infty}^{\infty} \frac{d \Omega(\lambda)}{\lambda-z}
$$


where $\Omega_{m, n}=\left\langle e_{m} \otimes \delta_{0}, P_{E} e_{n} \otimes \delta_{0}\right\rangle, m, n=1,2$ are the elements of the spectral matrix $\Omega$ of $H_{v, 0}$. Since $e_{1} \otimes \delta_{0}=\left(\begin{array}{c}\delta_{0} \\ 0\end{array}\right), e_{2} \otimes \delta_{0}=\left(\begin{array}{c}0 \\ \delta_{0}\end{array}\right)$ are cyclic vectors in $l^{2}\left(\mathbb{Z}_{+}, \mathbb{C}^{2}\right)$, it is sufficient to deal with this spectral measure, since any other will be absolutely continuous with respect to $\left(\Omega_{m, n}\right)_{m, n=1}^{2}$.

Explicitly, we have

$$
\mathcal{G}(z)=\frac{1}{c}\left(\begin{array}{cc}
m(z) & -1+m(z) \frac{m c^{2}-z+V_{0}}{c} \\
-1+m(z) \frac{m c^{2}-z+V_{0}}{c} & \frac{m c^{2}-z+V_{0}}{c}\left(-1+m(z) \frac{m c^{2}-z+V_{0}}{c}\right)
\end{array}\right),
$$

and the imaginary part of $\mathcal{G}(z)$ is

$$
\Im \mathcal{G}(z)=\frac{1}{c}\left(\begin{array}{cc}
\Im m(z) & -\frac{\Im z}{c} \mathfrak{R} m(z)+\Re a \Im m(z) \\
-\frac{\Im z}{c} \Re m(z)+\Re a \Im m(z) & -\frac{\Im z}{c}+\Re m(z) \Im a^{2}+\Im m(z) \Re a^{2}
\end{array}\right),
$$

with $a \equiv\left(m c^{2}-z+V_{0}\right) / c$. Hence, we conclude that the most important spectral properties of $H_{v, 0}(m, c)$ depend on the boundary behavior of the function $\Im m(E+i \varepsilon)$ as $\varepsilon \downarrow 0$ (since $\lim _{\varepsilon \downarrow 0} \Im a$ $=\lim _{\varepsilon \downarrow 0} \Im z=0$ ).

It follows from the definition of $m(z)$ and Theorem 3.1 in Chapter 9 of Ref. 3 that

$$
m(z)=\int_{-\infty}^{\infty} \frac{d \rho(\lambda)}{\lambda-z},
$$

with $\rho(\lambda)$ denoting a nondecreasing function of bounded variation, continuous to the right and

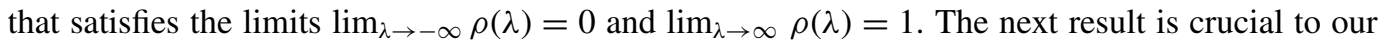
analysis.

Proposition 2.3: The spectral matrix measure $\Omega$ is absolutely continuous with respect to the Borel-Stieltjes measure $\rho$. Furthermore, there exists a symmetric and non-negative matrix $Y(E)$ such that

$$
d \Omega(E)=Y(E) d \rho(E)
$$

with

$$
Y(E)=\frac{1}{c}\left(\begin{array}{cc}
1 & \frac{m c^{2}-E+V_{0}}{c} \\
\frac{m c^{2}-E+V_{0}}{c} & \left(\frac{m c^{2}-E+V_{0}}{c}\right)^{2}
\end{array}\right) .
$$

Proof: By the Radon-Nikodym theorem, $\Omega$ is absolutely continuous with respect to $\rho$ if, and only if, there exists a symmetric integrable matrix $Y(E)$ such that (2.8) is valid for every $E$. Moreover, the elements of the matrix $Y(E)$ are given by

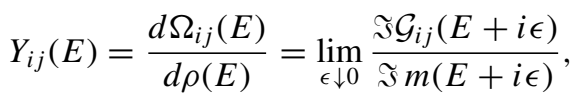

where the last equality comes from the Borel-Stieltjes inversion formula applied to $\Omega$ and $\rho$ (see the Appendix of Ref. 30).

We obtain (2.9) simply plugging (2.6) into (2.10). Observe that $Y(E)$ is a symmetric integrable matrix (i.e., $Y(E) \in \mathrm{L}^{1}(\mathbb{R}, d \rho)$ ). This concludes the proof of the proposition.

Proposition 2.3 shows that the most important features of the spectral matrix measure $\Omega$, such as its continuity or singularity with respect to Lebesgue and Hausdorff measures, are entirely determined by the Borel-Stieltjes measure $\rho$.

To deal with the spectral multiplicity issues, we need the following

Definition 2.4: The sesquilinear form

$$
\langle\mathbf{f}, \mathbf{g}\rangle=\int_{\mathbb{R}} \sum_{i, j=1}^{2} f_{i}^{*}(E) g_{j}(E) d \Omega_{i j}(E),
$$


defined for $f_{i}, g_{i} \in \mathrm{L}^{2}(\mathbb{R}, \mathbb{C}, d \rho)$ is non-negative provided $\|\mathbf{f}\|^{2}=\langle\mathbf{f}, \mathbf{f}\rangle \geq 0$ holds in this space.

Let us consider the sesquilinear form (2.11). Suppose that $\mathbf{f}$ is a simple function, that is, $\mathbf{f}(E)=\sum_{l=1}^{n} \chi_{B_{l}}(E)\left(f_{l, 1}, f_{l, 2}\right)$, where $\left(f_{l, 1}, f_{l, 2}\right) \in \mathbb{C}^{2}$ and $B_{l}$ are disjoint Borel sets. For this class of functions, (2.11) is a non-negative sesquilinear form. If $\mathbf{f}$ is such that $f_{i} \in \mathrm{L}^{2}(\mathbb{R}, \mathbb{C}, d \rho)$, we can approximate $\mathbf{f}$ by simple functions in order to obtain $\|\mathbf{f}\| \geq 0$. We have, as a consequence, a separable Hilbert space $\mathrm{L}^{2}\left(\mathbb{R}, \mathbb{C}^{2}, d \Omega\right)$ with scalar product (2.11).

Next, since the operator $H_{v, 0}(m, c)$ satisfies Weyl's limit point case (see also Ref. 30), there exists a unitary transformation $\tilde{U}: l^{2}\left(\mathbb{Z}_{+}, \mathbb{C}^{2}\right) \longrightarrow \mathrm{L}^{2}\left(\mathbb{R}, \mathbb{C}^{2}, d \Omega\right)$ such that $H_{v, 0}(m, c)$ $=\tilde{U}^{-1} \tilde{H}_{v, 0}(m, c) \tilde{U}$, where $\tilde{H}_{v, 0}(m, c): \mathrm{L}^{2}\left(\mathbb{R}, \mathbb{C}^{2}, d \Omega\right) \longrightarrow \mathrm{L}^{2}\left(\mathbb{R}, \mathbb{C}^{2}, d \Omega\right)$ is a multiplication operator (see, e.g., Theorem 2.12 of Ref. 30).

As for Schrödinger operators, we have the following result, whose proof is a direct extension of Lemma B.13 of Ref. 30:

Lemma 2.5: The set $\sigma(\rho):=\{E \in \mathbb{R}: \rho((E-\varepsilon, E+\varepsilon))>0$ for all $\varepsilon>0\}$ is precisely the spectrum $\sigma\left(\tilde{H}_{v, 0}(m, c)\right)$ of the multiplication operator $\tilde{H}_{v, 0}(m, c) \mathbf{f}(E)=E \mathbf{f}(E)$, with domain $\mathcal{D}\left(\tilde{H}_{v, 0}(m, c)\right)=\left\{\mathbf{f} \in \mathrm{L}^{2}\left(\mathbb{R}, \mathbb{C}^{2}, d \Omega\right) \mid E \mathbf{f}(E) \in \mathrm{L}^{2}\left(\mathbb{R}, \mathbb{C}^{2}, d \Omega\right)\right\}$.

Next, there exists a measurable unitary matrix $F(E)$ which diagonalizes $Y(E)$; that is,

$$
Y(E)=F^{-1}(E)\left(\begin{array}{cc}
y_{1}(E) & 0 \\
0 & y_{2}(E)
\end{array}\right) F(E),
$$

where $y_{i}(E), i=1,2$, are the integrable eigenvalues of $Y(E)$. The matrix $F(E)$ provides a unitary operator

$$
\mathrm{L}^{2}\left(\mathbb{R}, \mathbb{C}^{2}, d \Omega\right) \rightarrow \mathrm{L}^{2}\left(\mathbb{R}, y_{1} d \rho\right) \oplus \mathrm{L}^{2}\left(\mathbb{R}, y_{2} d \rho\right), \quad \mathbf{f}(E) \mapsto F \mathbf{f}(E),
$$

which leaves $\tilde{H}_{v, 0}(m, c)$ invariant (with respect to the scalar product defined in $\mathrm{L}^{2}\left(\mathbb{R}, \mathbb{C}^{2}, d \Omega\right)$ ). This permits us to investigate the spectral multiplicity of $\tilde{H}_{v, 0}(m, c)$.

Lemma 2.6: The spectral multiplicity of $\tilde{H}_{v, 0}(m, c)$ is 1 .

Proof: Evaluating $y_{1}(E)$ and $y_{2}(E)$ explicitly, we obtain $y_{1}(E)=0$ and $y_{2}(E)=\left(1+a^{2}\right) / c$, where $a=\left(m c^{2}-E+V_{0}\right) / c$. Thus,

$$
\mathrm{L}^{2}\left(\mathbb{R}, \mathbb{C}^{2}, d \Omega\right)=\{0\} \oplus \mathrm{L}^{2}\left(\mathbb{R}, y_{2} d \rho\right)=\mathrm{L}^{2}\left(\mathbb{R}, y_{2} d \rho\right) .
$$

Hence, $\tilde{H}_{v, 0}(m, c)$ is equivalent to multiplication by $E$ on $\mathrm{L}^{2}(\mathbb{R}, d \rho)$, since $y_{2}(E) d \rho(E)$ and $d \rho(E)$ are mutually absolutely continuous.

Remark 2.7: It is possible to show that we obtain similar results for the operator $H_{v, \phi}(m, c)$, i.e., with boundary condition (1.4). For this problem, we must adopt as a basis of the space of solutions the functions $u^{\phi}(z)$ and $u^{\phi^{*}}(z)$ (with $\phi^{*}=\phi+\pi / 2$ ), which satisfy the initial conditions

$$
\begin{array}{ll}
u_{2,-1}^{\phi}=\sin \phi, & u_{1,0}^{\phi}=\cos \phi, \\
u_{2,-1}^{\phi^{*}}=\cos \phi, & u_{1,0}^{\phi^{*}}=-\sin \phi .
\end{array}
$$

The result of Lemma 2.5 extends naturally, as well as the result of Proposition 2.3, except for the Radon-Nikodym derivative, now given by

$$
Y(E)=\frac{1}{c}\left(\begin{array}{cc}
\cos ^{2} \phi & \cos \phi\left(\frac{m c^{2}-E+V_{0}}{c}+\cos \phi \sin \phi\right) \\
\cos \phi\left(\frac{m c^{2}-E+V_{0}}{c}+\cos \phi \sin \phi\right) & \left(\frac{m c^{2}-E+V_{0}}{c} \cos \phi+\sin \phi\right)^{2}
\end{array}\right) ;
$$

the same situation holds for Lemma 2.6, with the eigenvalues of (2.12) given now by $y_{1}=0$ and $y_{2}=\left[\cos ^{2} \phi+\left(\left(m c^{2}-E+V_{0}\right) / c+\sin \phi\right)^{2}\right] / c$. 


\section{B. The essential spectrum}

Before we deal with the essential spectrum of $H_{v, \phi}(m, c)$, it would be interesting to characterize the spectrum of the free operator $H_{0,0}(m, c)$ (Ref. 8) and its spectral matrix measure.

Proposition 2.8: The spectrum of the free operator is given by the intervals

$$
\sigma\left(H_{0,0}(m, c)\right)=\left[-\sqrt{m^{2} c^{4}+4 c^{2}},-m c^{2}\right] \cup\left[m c^{2}, \sqrt{m^{2} c^{4}+4 c^{2}}\right] .
$$

Moreover, the spectral matrix measure $\Omega$ is purely absolutely continuous (with respect to the Lebesgue measure $\ell$ ) in these intervals.

Proof: In order to prove this proposition, we will determine the exact behavior of the function $m(E+i \varepsilon)$ as $\varepsilon \downarrow 0$.

After some manipulations, it follows by (1.3) that

$$
\left(-\Delta-\frac{m^{2} c^{4}-z^{2}}{c^{2}}\right) \psi_{j, n}=0, \quad n \in \mathbb{Z}_{+},
$$

where $\psi_{j}, j=1,2$, are the components of the spinor $\Psi$.

Since $m(z)$ is uniquely defined imposing that $\Psi=-u^{N}+m(z) u^{D}$ is $l^{2}\left(\mathbb{Z}_{+}, \mathbb{C}^{2}\right)$, it is simple to obtain

$$
m(z)=-\frac{w}{2}+\sqrt{\frac{w^{2}}{4}-1},
$$

with $w=\left[m^{2} c^{4}-z^{2}+2 c^{2}\right] / c^{2}$. Now, put

$$
\Im m(E)=\limsup _{\varepsilon \downarrow 0} \Im m(z), \quad z=E+i \varepsilon,
$$

and let $L(\rho)$ be the set of all $E \in \mathbb{R}$ for which this limit exists. It is known (see Appendix B of Ref. 30) that the minimal (or essential) supports $\mathcal{M}, \mathcal{M}_{\mathrm{ac}}$, and $\mathcal{M}_{\mathrm{s}}$ of $\rho$, the absolutely continuous part $\rho_{\mathrm{ac}}$ and the singular part $\rho_{\mathrm{s}}$ of $\rho$, with respect to the Lebesgue measure in $\mathbb{R}$, are, respectively, given by $E \in L(\rho)$ such that $0<\Im m(E) \leq \infty, 0<\Im m(E)<\infty$ and $\Im m(E)=\infty$. These criteria can be obtained by de la Vallée-Poussin's decomposition theorem, ${ }^{24}$ the Radon-Nikodym theorem and the following application of Lemma 3 in Ref. 11.

Lemma 2.9: If the Radon-Nikodym derivative $(d \rho / d \ell)(E)$ exists and takes values in $[0, \infty]$, then $\Im m(E)$ also exists and $(d \rho / d \ell)(E)=(1 / \pi) \Im m(E)(\ell$ is the Lebesgue measure on $\mathbb{R})$.

Since

$$
\lim _{\varepsilon \downarrow 0} \Im m(E+i \varepsilon)= \begin{cases}\frac{\sqrt{\left(E^{2}-m^{2} c^{4}\right)\left(m^{2} c^{4}+4 c^{2}-E^{2}\right)}}{2 c^{2}}, & \text { if } E^{2} \in\left[m^{2} c^{4}, m^{2} c^{4}+4 c^{2}\right], \\ 0, & \text { otherwise, }\end{cases}
$$

it follows by the above criteria and Proposition 2.3 that the spectral matrix measure $\Omega$ is purely absolutely continuous on the interval defined by (2.13); moreover, the essential spectrum of $H_{0,0}(m, c)$ coincides with its own spectrum. This conclude the proof of the proposition.

Remark 2.10: As we have discussed in Remark 1.2, the operator $H_{v, \phi}(m, c)$ with boundary condition (1.4) at $n=-1$ may be written as a rank-one perturbation of $H_{v, 0}(m, c)$; hence, $\sigma_{\text {ess }}\left(H_{v, \phi}(m, c)\right)=\sigma_{\text {ess }}\left(H_{v, 0}(m, c)\right)$, by Weyl's criterion (see, for instance, Sec. 11.3 of Ref. 6). Thus, it is sufficient to deal with $H_{v, 0}(m, c)$ in order to determine the essential spectrum of $H_{v, \phi}(m, c)$.

We are now ready to prove Theorem 1.3.

Proof: (Theorem 1.3) By taking into account the above results and Remark 2.10, the proof of Theorem 1.3 reduces to a direct extension of Theorem 3.13 in Ref. 5 to the discrete Dirac operator.

The eigenvalues of $H_{0,0}^{\prime}(m, c)$ are isolated points of the essential spectrum of $H_{v, 0}(m, c)$; hence, they cannot belong to the continuous spectrum of $H_{v, 0}(m, c)$, neither be eigenvalues of infinite 
multiplicity (since we have a unidimensional problem). Thus, they must be accumulation points of the discrete spectrum of $H_{v, 0}(m, c)$.

Since the operator $H_{0,0}^{\prime}(m, c)$ is a rank-one perturbation of $H_{0,0}(m, c)$, it follows that its spectrum is the union of the interval (2.13) with the possible addition of a finite number of isolated points. Thus, it follows by Theorem 1.3 that the essential spectrum of $H_{v, \phi}(m, c)$ has the same structure.

\section{TRANSFER MATRICES AND PRÜFER VARIABLES}

In order to determine the exact Hausdorff dimension of the spectral matrix measure $\Omega$ and, consequently, the spectral nature of $H_{v, \phi}(m, c)$, we study the exact asymptotic behavior of the solutions to the Dirac eigenvalue equation

$$
H_{v, \phi}(m, c) \Psi=E \Psi
$$

with $E \in \mathbb{R}$; this is an important step in our approach. It is here that the concepts of transfer matrix and Prüfer variables play a fundamental role. What follows is an adaptation of the material presented in Sects. 2 and 4 of Ref. 20 to the Dirac operator setting.

For $E \in \mathbb{C}$, let

$$
T(n, n-1 ; E)=\left(\begin{array}{cc}
1+\frac{m^{2} c^{4}-\left(E-V_{n}\right)^{2}}{c^{2}} & \frac{m c^{2}+E-V_{n}}{c} \\
\frac{m c^{2}-E+V_{n}}{c} & 1
\end{array}\right)
$$

be the $2 \times 2$ transfer matrix associated with the $l^{2}\left(\mathbb{Z}_{+}, \mathbb{C}^{2}\right)$ solution to the Dirac equation (3.1) (see Refs. 7 and 8).

The equation

$$
\left(\begin{array}{c}
\psi_{1, n+1} \\
\psi_{2, n}
\end{array}\right)=T(n, n-1 ; E)\left(\begin{array}{c}
\psi_{1, n} \\
\psi_{2, n-1}
\end{array}\right)
$$

holds for $n \geq 0$, with $\left(\begin{array}{c}\psi_{1,0} \\ \psi_{2,-1}\end{array}\right)=\left(\begin{array}{c}\cos \phi \\ \sin \phi\end{array}\right)$ satisfying (1.4) for some $\phi \in[0, \pi]$. Another important tool is the product of the $n+1$ first transfer matrices, denoted by

$$
T(n ; E)=T(n, n-1 ; E) T(n-1, n-2 ; E) \ldots T(0,-1 ; E) .
$$

Given the values (1.5) of the potential $V_{n}$ and the sparseness condition (1.7), only two different $2 \times 2$ matrices appear on the rhs of (3.3): that is,

$$
T_{v}(E)=\left(\begin{array}{cc}
1+\frac{m^{2} c^{4}-(E-v)^{2}}{c^{2}} & \frac{m c^{2}+E-v}{c} \\
\frac{m c^{2}-E+v}{c} & 1
\end{array}\right)
$$

and

$$
T_{0}(E)=\left(\begin{array}{cc}
1+\frac{m^{2} c^{4}-E^{2}}{c^{2}} & \frac{m c^{2}+E}{c} \\
\frac{m c^{2}-E}{c} & 1
\end{array}\right)
$$

occur depending on the entry $n$ in (3.2) being or not $a_{j}^{\omega} \in \mathcal{A}$.

Let $E= \pm \sqrt{m^{2} c^{4}+2 c^{2}(1-\cos \varphi)}$, with $\varphi \in[0, \pi)$, be parametrizations of the intervals (2.13). Now note that, for such energies, the free matrix $T_{0}(E)$ is similar to a purely clockwise rotation 
$R(\varphi)$, that is,

$$
U T_{0}(E) U^{-1}=\left(\begin{array}{cc}
\cos \varphi & \sin \varphi \\
-\sin \varphi & \cos \varphi
\end{array}\right)=R(\varphi)
$$

where

$$
U \equiv\left(\begin{array}{cc}
-\frac{m c^{2}-E}{c \sin \varphi} & -\frac{1-\cos \varphi}{\sin \varphi} \\
0 & 1
\end{array}\right)
$$

Note also that $U$ is not uniquely defined since any other matrix $U^{\prime}=H U$, with $H$ commuting with $R$, satisfies (3.4).

Since the product of rotation matrices is also a rotation, we obtain

$$
U T(n ; E) U^{-1}=R\left(\left(n-a_{k}^{\omega}\right) \varphi\right) P(E) R\left(\left(a_{k}^{\omega}-a_{k-1}^{\omega}\right) \varphi\right) \cdots P(E) R\left(\left(a_{1}^{\omega}+1\right) \varphi\right)
$$

as the conjugation of (3.3) by $U^{-1}$, for every $n \in \mathbb{N}$ and $\omega_{j} \in\left\{0,, 1,2, \ldots,\left[j^{\eta}\right]\right\}, j \geq 1 ; k$ is an integer such that $a_{k}^{\omega} \leq n<a_{k+1}^{\omega} ; P(E)$ is defined by

$$
\begin{aligned}
P(E) R(\varphi) & =U T_{v}(E) U^{-1} \\
& =\left(\begin{array}{cc}
1+\frac{v E_{+}}{2 c^{2}}-\frac{v^{2}}{c^{2}} & -\frac{v}{2 c^{2}} \sqrt{\frac{E_{+} E_{-}}{F}}\left(E_{+}-\frac{4 c^{2}}{E_{+}}-2 v\right) \\
-\frac{v}{2 c^{2}} \sqrt{\frac{E_{+} F}{E_{-}}} & 1-\frac{v E_{+}}{2 c^{2}}
\end{array}\right),
\end{aligned}
$$

where $E_{+}=E+m c^{2}, E_{-}=E-m c^{2}$, and $F=m^{2} c^{4}+4 c^{2}-E^{2}$.

Next we consider the following change of variables, known as EFGP transform:

$$
\mathbf{v}_{\mathbf{k}}:=\left(\begin{array}{c}
R_{k} \cos \theta_{k}^{\omega} \\
R_{k} \sin \theta_{k}^{\omega}
\end{array}\right)=U\left(\begin{array}{c}
\psi_{1, k} \\
\psi_{2, k-1}
\end{array}\right)=\left(\begin{array}{c}
\frac{\left(E-m c^{2}\right) \psi_{1, k} / c-(1-\cos \varphi) \psi_{2, k-1}}{\sin \varphi} \\
\psi_{2, k-1}
\end{array}\right),
$$

The variables $R_{k}$ and $\theta_{k}^{\omega}$ are called, respectively, Prüfer radii and angles. Observe that the Prüfer angles are random variables, since $\omega_{j}$ are randomly distributed. The Prüfer radii, on the other hand, are random variables only as a function of the Prüfer angles, and their dependence on $\omega$ will be omitted.

Remark 3.1: The convention we have employed here differs from the convention in Ref. 14, since the functions $\cos \theta_{k}$ and $\sin \theta_{k}$ are exchanged. Nevertheless, the behavior of the Prüfer variables are identical in both conventions.

We nonetheless use a slightly different expression of (3.7), more adequate to relation (3.5). Given the vectors

$$
\mathbf{v}_{k}=\left(R_{k-1} \cos \theta_{k}^{\omega}, R_{k-1} \sin \theta_{k}^{\omega}\right), \quad \tilde{\mathbf{v}}_{k}=\left(R_{k} \cos \tilde{\theta}_{k}^{\omega}, R_{k} \sin \tilde{\theta}_{k}^{\omega}\right),
$$

the Prüfer variables $\left(R_{k}, \theta_{k}^{\omega}\right)_{k \geq 0}$ satisfy a recurrence relation induced by

$$
\mathbf{v}_{k}=R\left(\left(a_{k}^{\omega}-a_{k-1}^{\omega}\right) \varphi\right) \tilde{\mathbf{v}}_{k-1}
$$

and

$$
\tilde{\mathbf{v}}_{k}=P(E) \mathbf{v}_{k},
$$

with $\mathbf{v}_{1}=R\left(\left(a_{1}^{\omega}+1\right) \varphi\right) \tilde{\mathbf{v}}_{0}$,

$$
\begin{gathered}
\tilde{\mathbf{v}}_{0}\left(\theta_{0}\right)=R_{0}\left(\begin{array}{c}
\cos \theta_{0} \\
\sin \theta_{0}
\end{array}\right)=U\left(\begin{array}{c}
\cos \phi \\
\sin \phi
\end{array}\right)=\left(\begin{array}{c}
\frac{\left(E-m c^{2}\right) / c \cos \phi-(1-\cos \varphi) \sin \phi}{\sin \varphi} \\
\cos \phi
\end{array}\right), \\
R_{0}^{2}=\left(\frac{\left(\left(E-m c^{2}\right) \cos \phi\right) / c-(1-\cos \varphi) \sin \phi}{\sin \varphi}\right)^{2}+\cos ^{2} \phi .
\end{gathered}
$$


Thus, if $\psi_{n}=\left(\psi_{1, n}, \psi_{2, n-1}\right)$ represents a solution to (3.1) satisfying the initial conditions $\mathbf{u}_{0}=(\cos \phi, \sin \phi)$, then

$$
R(\varphi) \tilde{\mathbf{v}}_{k}=U \mathbf{u}_{a_{k}+1} .
$$

By equivalence of norms, the growth of $T(n ; E)$ may be controlled by the euclidean norm (see Sec. 4 of Ref. 20 for details)

$$
\left\|U T(n ; E) U^{-1} v_{0}\right\|^{2}=\left\|U T\left(a_{N}^{\omega}+1 ; E\right) U^{-1} v_{0}\right\|^{2}=R_{N}^{2},
$$

where the equality holds for any unit vector $v_{0}=\left(\cos \theta_{0}, \sin \theta_{0}\right)$ and for each $n$ such that $a_{N}^{\omega} \leq n$ $<a_{N+1}^{\omega}$. Thus, from Eqs. (3.8), (3.9), and (3.6), $R_{N}^{2}$ can be written as

$$
\left(R_{N}\right)^{2}=\left(R_{0}\right)^{2} \prod_{n=1}^{N}\left(\frac{R_{n}}{R_{n-1}}\right)^{2}=\left(R_{0}\right)^{2}\left(\exp \left\{\frac{1}{N} \sum_{n=1}^{N} \ln f\left(\theta_{n}^{\omega}, \varphi\right)\right\}\right)^{N} .
$$

with

$$
f\left(\theta^{\omega}, \varphi\right):=\left(A(\varphi) \cos \theta^{\omega}+B(\varphi) \sin \theta^{\omega}\right)^{2}+\left(C(\varphi) \cos \theta^{\omega}+D(\varphi) \sin \theta^{\omega}\right)^{2},
$$

where $A(\varphi)=1+\frac{v\left(E+m c^{2}\right)}{2 c^{2}}-\frac{v^{2}}{c^{2}}, D(\varphi)=1-\frac{v\left(E+m c^{2}\right)}{2 c^{2}}$,

$$
B(\varphi)=-\frac{v}{2 c^{2}} \sqrt{\frac{E^{2}-m^{2} c^{4}}{m^{2} c^{4}+4 c^{2}-E^{2}}}\left(E+m c^{2}-\frac{4 c^{2}}{E+m c^{2}}-2 v\right),
$$

and

$$
C(\varphi)=-\frac{v}{2 c^{2}} \sqrt{\frac{\left(E+m c^{2}\right)\left(m^{2} c^{4}+4 c^{2}-E^{2}\right)}{E-m c^{2}}}
$$

are the entries of $P(E)$.

The Prüfer angles $\left(\theta_{k}^{\omega}\right)_{k \geq 1}$ are, on the other hand, obtained recursively by

$$
\theta_{k}^{\omega}=\tan ^{-1}\left(\frac{C+D \tan \theta_{k-1}^{\omega}}{A+B \tan \theta_{k-1}^{\omega}}\right)-\left(\beta^{k}+\omega_{k}-\omega_{k-1}\right) \varphi
$$

for $k>1$, with $\theta_{1}^{\omega}$ given by $\theta_{1}^{\omega}=\theta_{0}-\left(a_{1}+\omega_{1}\right) \varphi$.

Hence, the determination of the exact asymptotic behavior of the sequence $\left(R_{n}\left(\theta_{0}\right)\right)_{n \geq 1}$ involves an estimate of the Birkhoff-like sum

$$
\frac{1}{N} \sum_{n=1}^{N} \ln f\left(v, \theta_{n}^{\omega}\right)
$$

for $N$ large, which, on the other hand, depends on the distribution properties of the sequence $\left(\theta_{n}^{\omega}\right)_{n>1}$ of the Prüfer angles. The tool that intertwines these elements is the following theorem (recall that a sequence $w=\left(x_{n}\right)_{n \geq 1}$ is said to be uniformly distributed modulo $\pi$ (u.d. $\bmod \pi$ ) if it is equally distributed, in fractional portions, over half open subintervals of $[0, \pi)$ ).

Theorem 3.2 (Theorem 1.1 in Ref. 15): The sequence $w=\left(x_{n}\right)_{n \geq 1}$ of real numbers $x_{n} \in[0, \pi)$ is u. d. $\bmod \pi$, if and only if, for every continuous real function $h$ defined on the closed interval $I=[0, \pi]$, we have

$$
\lim _{N \rightarrow \infty} \frac{1}{N} \sum_{n=1}^{N} h\left(\left\{x_{n}\right\}\right)=\frac{1}{\pi} \int_{0}^{\pi} h(x) d x .
$$

Theorem 3.2 provides a criterion that permits the substitution, on the asymptotic limit $N \rightarrow \infty$, of the average (3.13) by the integral

$$
\frac{1}{\pi} \int_{0}^{\pi} \ln f(v, \theta) d \theta
$$


in case the sequence $\left(\theta_{n}^{\omega}\right)_{n \geq 1}$ of the Prüfer angles is u. d. $\bmod \pi$ and $\ln f(v, \theta)$, with $f(v, \theta)$ given by (3.11), is a periodic Riemann integrable function of period $\pi$.

Lemma 3.3: The function $h(\theta):=\ln f(v, \theta)$ is a periodic Riemann integrable function of period $\pi$, whose average is given by

$$
\frac{1}{\pi} \int_{0}^{\pi} h(\theta) d \theta=\ln r(v, E)
$$

with

$$
r(v, E)=1+\frac{1}{\left(m^{2} c^{4}+4 c^{2}-E^{2}\right)} \frac{v^{2}}{c^{2}}\left[\frac{\left(E^{2}-m^{2} c^{4}\right)^{2}+4 m^{2} c^{6}}{\left(E^{2}-m^{2} c^{4}\right)}-4 v E+2 v^{2}\right] .
$$

Proof: The proof of the lemma is an adaptation of some results presented in Sec. IV in Ref. 20.

Lemma 3.3 and Theorem 3.2 provide a precise estimate for the asymptotic limit of the "time average" (3.13) under the hypothesis of the u. d. $\bmod \pi$ of the sequence $\left(\theta_{n}^{\omega}\right)_{n \geq 1}$ of Prüfer angles.

Lemma 3.4: Let $\left(R_{n}\left(\theta_{0}\right)\right)_{n \geq 1}$ be the sequence of the Prüfer radii which satisfy the initial conditions $\mathbf{v}_{0}=(\cos \vartheta, \sin \vartheta)$. Suppose there is a set $A \subset \mathbb{R}$ of null Lebesgue measure so that the sequence $\left(\theta_{n}^{\omega}\right)_{n \geq 1}$ of the Prüfer angles is $\mathrm{u}$. d. $\bmod \pi$ for $\varphi \in[0, \pi) \backslash A$. Then,

$$
C_{N}^{-1} r^{N} \leq\left(R_{N}\left(\theta_{0}\right)\right)^{2} \leq C_{N} r^{N}
$$

where $C_{N}$ is a real number such that $C_{N}>1$ and $\lim _{N \rightarrow \infty} C_{N}^{1 / N}=\left(R_{0}\right)^{2}$, with $r$ given by (3.16).

Proof: The inequalities (3.17) follow from the hypotheses of the lemma, by Eqs. (3.10) and (3.15) and the estimate

$$
\left|\frac{1}{N} \sum_{n=1}^{N} \ln f\left(v, \theta_{n}^{\omega}\right)-\frac{1}{\pi} \int_{0}^{\pi} \ln f(v, \theta) d \theta\right| \leq C D_{N}^{*},
$$

where

$$
D_{N}^{*}(\theta)=D_{N}^{*}\left(\theta_{1}, \ldots, \theta_{N}\right)=\sup _{0<\vartheta \leq \pi}\left|\frac{\operatorname{card}\left(\left\{k: \theta_{k} \bmod \pi \in[0, \theta), 1 \leq k \leq N\right\}\right)}{N}-\vartheta\right|
$$

is the so-called discrepancy of the sequence $\left(\theta_{n}\right)_{n \geq 1}$ (see Ref. 15 for an ample discussion on discrepancy) and $C$ is some positive constant. For the second part of the lemma, we need

Theorem 3.5 (Corollary 1.1 of Chapter 2 in Ref. 15): A sequence $w$ is u. d. $\bmod \pi$ if, and only if, $\lim _{N \rightarrow \infty} D_{N}^{*}(w)=0$.

It follows by the hypothesis of uniform distribution and Theorem 3.5 that

$$
\lim _{N \rightarrow \infty} D_{N}^{*}\left(\theta^{\omega}\right)=0
$$

hence, $\lim _{N \rightarrow \infty} C_{N}^{1 / N}=\left(R_{0}\left(\theta_{0}\right)\right)^{2}$, and the proof of Lemma 3.4 is complete.

Now we finally deal with the uniform distribution of the sequence $\left(\theta_{n}^{\omega}\right)_{n \geq 1}$.

Theorem 3.6: The sequence of Prüfer angles $\left(\theta_{n}^{\omega}\right)_{n \geq 1}$ is $\mathrm{u}$. d. $\bmod \pi$ for all $\varphi / \pi \in[0,1] \backslash \mathbb{Q}$ and all $\omega \in \Lambda$, apart from a set with null $v$ measure.

Proof: The proof is exactly the same of Theorem 3.2 in Ref. 2. 


\section{HAUSDORFF DIMENSION OF THE SPECTRAL MEASURE}

This section is devoted to the determination of the Hausdorff dimension of the spectral measure of $H_{v, \phi}(m, c)$. At the end, the proofs of Theorems 1.4 and 1.5 are presented.

\section{A. Basic definitions and generalized subordinacy theory}

First, we recall some useful definitions. An almost complete description is found in Ref. 17. Given a Borel set $S \subset \mathbb{R}$ and $\alpha \in[0,1]$, consider the number

$$
Q_{\alpha, \delta}(S)=\inf \left\{\sum_{\nu=1}^{\infty}\left|b_{\nu}\right|^{\alpha}:\left|b_{\nu}\right|<\delta ; S \subset \bigcup_{\nu=1}^{\infty} b_{\nu}\right\},
$$

with the infimum taken over all covers by intervals of size at most $\delta$. The limit

$$
h^{\alpha}(S)=\lim _{\delta \downarrow 0} Q_{\alpha, \delta}(S),
$$

is called $\alpha$-dimensional Hausdorff (outer) measure. The counting measure, at $\alpha=0$, and the Lebesgue measure, at $\alpha=1$, are important particular cases. It is clear by the definitions (4.1) and (4.2) that $h^{\alpha}(S)$ is an outer measure on $\mathbb{R}$ (see Ref. 10). For $\beta<\alpha<\gamma$,

$$
\delta^{\alpha-\gamma} Q_{\gamma, \delta}(S) \leq Q_{\alpha, \delta}(S) \leq \delta^{\alpha-\beta} Q_{\beta, \delta}(S),
$$

holds for any $\delta>0$ and $S \subset \mathbb{R}$. So, if $h^{\alpha}(S)<\infty$, then $h^{\gamma}(S)=0$ for $\gamma>\alpha$; if $h^{\alpha}(S)>0$, then $h^{\beta}(S)=\infty$ for $\beta<\alpha$. Thus, for every Borel set $S$, there is a unique $\alpha_{S}$ such that $h^{\alpha}(S)=0$ if $\alpha>\alpha_{S}$ and $h^{\alpha}(S)=\infty$ if $\alpha_{S}<\alpha$. The number $\alpha_{S}$ is called the Hausdorff dimension of the set $S$.

Another useful concept is the exact dimension of a measure, taken from Ref. 23.

Definition 4.1: A Borel measure $\mu$ in $\mathbb{R}$ is said to be of exact dimension $\alpha, \alpha \in[0,1]$, if two requirements hold: (1) for every $\beta \in[0,1]$ with $\beta<\alpha$ and $S$ a set of dimension $\beta, \mu(S)=0$ (which means that $\mu(S)$ gives zero weight to any set $S$ with $\left.h^{\alpha}(S)=0\right)$; (2) there is a set $S_{0}$ of dimension $\alpha$ which supports $\mu$ in the sense that $\mu\left(\mathbb{R} \backslash S_{0}\right)=0$.

Finally, we recall the notions of continuity and singularity of a measure with respect to the Hausdorff measure. Given $\alpha \in[0,1]$, a measure $\mu$ is called $\alpha$-continuous if $\mu(S)=0$ for every set $S$ with $h^{\alpha}(S)=0$; it is called $\alpha$-singular if it is supported on some set $S$ with $h^{\alpha}(S)=0$.

Remark 4.2: It is possible to reformulate Definition 4.1 in this context: a measure $\mu$ is said to have exact dimension $\alpha$ if, for every $\epsilon>0$, it is simultaneously $(\alpha-\epsilon)$-continuous and $(\alpha+\epsilon)$-singular.

Jitomirskaya and Last ${ }^{12}$ extended, to Hausdorff measures, the Gilbert-Pearson theory of subordinacy ${ }^{11}$ for Lebesgue measures, which relates the spectral property of $\rho$ to the rate of growth of the solutions to the Schrödinger equation. Now we describe the extension of these results to Dirac operators (1.2).

A solution $\Psi$ to (3.1) is said to be subordinate if

$$
\lim _{l \rightarrow \infty} \frac{\|\Psi\|_{l}}{\|\Phi\|_{l}}=0
$$

holds for any linearly independent solution $\Phi$ to (3.1), where $\|\cdot\|_{l}$ denotes the $l^{2}\left(\mathbb{Z}_{+}, \mathbb{C}^{2}\right)$-norm truncated at the length $l \in \mathbb{R}$, i.e.,

$$
\|\Psi\|_{l}^{2} \equiv \sum_{n=0}^{[l]}\left[\left|\psi_{1, n}\right|^{2}+\left|\psi_{2, n}\right|^{2}\right]+(l-[l])\left(\left|\psi_{1,[l]+1}\right|^{2}+\left|\psi_{2,[l]+1}\right|^{2}\right),
$$

$[l]$ the integer part of $l$. 

equality

Following Jitomirskaya-Last, ${ }^{12}$ for any given $\epsilon>0$ introduce a length $l(\epsilon) \in(0, \infty)$ by the

$$
\left\|u^{D}\right\|_{l(\epsilon)}\left\|u^{N}\right\|_{l(\epsilon)}=\frac{c}{2 \epsilon}
$$

(see Eq. (1.12) in Ref. 12), where $u^{D}$ and $u^{N}$ are the solutions to (3.1) which satisfy the initial conditions (2.4).

At most one of the solutions $\left\{u^{D}, u^{N}\right\}$ to (3.1) belongs to $l^{2}\left(\mathbb{Z}_{+}, \mathbb{C}^{2}\right)$, thanks to the constancy of the Wronskian, which follows by the Green's identity; that is, for $n \geq-1$,

$$
\sum_{n=0}^{N}\left(\Psi_{n}^{*}\left(H_{D} \Phi\right)_{n}-\left(H_{D} \Psi\right)_{n}^{*} \Phi_{n}\right)=W[\Phi, \Psi](N)-W[\Phi, \Psi](-1)=0
$$

(where $W[\Phi, \Psi](n)=c\left(\phi_{1, n+1} \psi_{2, n}^{*}-\phi_{2, n} \psi_{1, n+1}^{*}\right)$; see also Chapter 9 in Ref. 3 ). Hence, the lefthand side of (4.3) is a monotone increasing function of $l$ which vanishes at $l=0$ and diverges as $l \rightarrow \infty$. On the other hand, the right-hand side of (4.3) is a monotone decreasing function of $\epsilon$ which diverges as $\epsilon \rightarrow 0$. It is then concluded that the function $l(\epsilon)$ is a well-defined monotone decreasing and continuous function of $\epsilon$ which diverges as $\epsilon \rightarrow 0$.

What follows is the version of Jitomirskaya-Last inequalities for the discrete Dirac operators.

Theorem 4.3: Let $H_{D}(m, c)$ be the Dirac operator (1.2) that satisfies the boundary condition (1.8). Given $\epsilon>0$, we obtain

$$
\frac{5-\sqrt{24}}{m(E+i \epsilon)} \leq \frac{\left\|u^{D}\right\|_{l(\epsilon)}}{\left\|u^{N}\right\|_{l(\epsilon)}} \leq \frac{5+\sqrt{24}}{m(E+i \epsilon)}
$$

In order to prove this theorem, we only need the following lemma, which is an adaptation of Lemma 3.1 in Ref. 12; with such result at hand, the proof of Theorem 4.3 follows the same lines as the proof of Theorem 1.1 in Ref. 12.

The function $\chi_{n}(z)$ in Lemma 4.4 is nothing but the unique $l^{2}\left(\mathbb{Z}_{+}, \mathbb{C}^{2}\right)$ solution of the Dirac equation (2.3), the one associated with the definition of $m(z)$ (see Sec. II).

Lemma 4.4 (Variation of Parameters): For every $n \geq 0, \chi_{n}(z)$ satisfies the identity

$$
\begin{aligned}
\left(\begin{array}{c}
\chi_{1, n+1}(z) \\
\chi_{2, n}(z)
\end{array}\right) & =-\left(\begin{array}{c}
u_{1, n+1}^{N}(E) \\
u_{2, n}^{N}(E)
\end{array}\right)+m(z)\left(\begin{array}{c}
u_{1, n+1}^{D}(E) \\
u_{2, n}^{D}(E)
\end{array}\right) \\
& -\frac{i \epsilon}{c}\left(\begin{array}{c}
u_{1, n+1}^{N}(E) \\
u_{2, n}^{N}(E)
\end{array}\right) \sum_{k=1}^{n}\left\{u_{1, k+1}^{D}(E) \chi_{1, k}(z)+u_{2, k}^{D}(E) \chi_{2, k}(z)\right\} \\
& +\frac{i \epsilon}{c}\left(\begin{array}{c}
u_{1, n+1}^{D}(E) \\
u_{2, n}^{D}(E)
\end{array}\right) \sum_{k=1}^{n}\left\{u_{1, k+1}^{N}(E) \chi_{1, k}(z)+u_{2, k}^{N}(E) \chi_{2, k}(z)\right\}
\end{aligned}
$$

Proof: We denote by $\left(\begin{array}{c}\tilde{v}_{1, n+1}(E) \\ \tilde{v}_{2, n}(E)\end{array}\right)$ the right-hand side of (4.4), and let $\left(\begin{array}{c}\tilde{v}_{1,0}(E) \\ \tilde{v}_{2,-1}(E)\end{array}\right)=\left(\begin{array}{c}m(z) \\ -1\end{array}\right)$. Regarding the Wronskian constancy, it is easy to verify that

$$
\left(\begin{array}{c}
\tilde{v}_{1, n+1}(E) \\
\tilde{v}_{2, n}(E)
\end{array}\right)=\left(\begin{array}{c}
\tilde{v}_{1, n}(E)+\frac{m c^{2}-V_{n}+E}{c} \tilde{v}_{2, n}(E)+\frac{i \epsilon}{c} \chi_{2, n}(z) \\
\tilde{v}_{2, n-1}(E)+\frac{m c^{2}-V_{n}-E}{c} \tilde{v}_{1, n}(E)-\frac{i \epsilon}{c} \chi_{1, n}(z)
\end{array}\right)
$$

holds for every $n \geq-1$. Since

$$
\left(\begin{array}{c}
\chi_{1, n+1}(z) \\
\chi_{2, n}(z)
\end{array}\right)=\left(\begin{array}{c}
\chi_{1, n}(z)+\frac{\left(m c^{2}-V_{n}+E+i \epsilon\right) \chi_{2, n}(z)}{c} \\
\chi_{2, n-1}(z)+\frac{\left(m c^{2}-V_{n}-E-i \epsilon\right) \chi_{1, n}(z)}{c}
\end{array}\right)
$$


and $\left(\begin{array}{c}\tilde{v}_{1,0}(E) \\ \tilde{v}_{2,-1}(E)\end{array}\right)=\left(\begin{array}{c}m(z) \\ -1\end{array}\right)=\left(\begin{array}{c}\chi_{1,0}(E) \\ \chi_{2,-1}(E)\end{array}\right)$, it follows by induction that $\tilde{v}_{n}(z)=\chi_{n}(z)$ for every $n \geq 0$.

It is a direct consequence of Theorem 4.3 that Theorem 1.2 in Ref. 12 and its corollaries also hold true: if $\rho$ is defined by (2.7), then, with $b=\alpha /(2-\alpha)$,

$$
D_{\rho}^{\alpha}(E):=\limsup _{\epsilon \downarrow 0} \frac{\rho((E-\epsilon, E+\epsilon))}{(2 \epsilon)^{\alpha}}=\infty
$$

if, and only if,

$$
\liminf _{l \rightarrow \infty} \frac{\left\|u^{D}\right\|_{l}}{\left\|u^{N}\right\|_{l}^{b}}=0
$$

and $D_{\rho}^{\alpha}(E)$ is the so-called Hausdorff upper derivative of $\rho$ at $E$ (see Ref. 17 for a detailed discussion of this concept).

Before we proceed, we need some important results regarding the behavior of the generalized eigenfunction $u^{D}$. Next we present a version of Theorem 3.10 in Ref. 19.

Proposition 4.5: Fix $\delta>0$. For almost every $E$ with respect to the measure $\rho$,

$$
\frac{1}{l} \sum_{n=0}^{l}\left(\left|u_{1, n}^{D}(E)\right|^{2}+\left|u_{2, n}^{D}(E)\right|^{2}\right) \leq C_{E}(\ln l)^{1+\delta}, \quad l \geq 2 .
$$

In particular, by taking $\delta=1$,

$$
\limsup _{l \rightarrow \infty} \frac{\left\|u^{D}(E)\right\|}{l^{1 / 2} \ln l}<\infty
$$

Proof: Define the function $g_{k}(E)=2^{-k} \sum_{n=0}^{2^{k}}\left(\left|u_{1, n}^{D}(E)\right|^{2}+\left|u_{2, n}^{D}(E)\right|^{2}\right)$. It follows from Proposition 3.3 in Ref. 19 (in fact, from a straightforward adaptation of this result) that $\int g_{k}(E) d \rho_{\mathrm{ac}}(E) \leq$ $C<\infty$; thus, $\sum_{k=0}^{\infty} k^{-1-\delta} g_{k}(E) \in \mathrm{L}^{1}(\mathbb{R}, d \rho)$. This implies the inequality $g_{k}(E) \leq \widetilde{C}_{E} k^{1+\delta}$ for almost every $E$ with respect to $\rho$. Let $2^{k-1} \leq l \leq 2^{k}$. Then

$$
\frac{1}{l} \sum_{n=0}^{l}\left(\left|u_{1, n}^{D}(E)\right|^{2}+\left|u_{2, n}^{D}(E)\right|^{2}\right) \leq 2 \widetilde{C}_{E} k^{1+\delta} \leq C_{E}(\ln l)^{1+\delta},
$$

with $C_{E}=2\left(1+\frac{1}{\ln 2}\right) \widetilde{C}_{E}$. In particular, by picking the value $\delta=1$, relation (4.6) follows from (4.5). This completes the proof of the proposition.

Lemma 4.6: Let $u^{D}(E)$ and $u^{N}(E)$ be the solutions to (3.1) that satisfy the initial conditions (2.4). Then

$$
\left\|u^{D}(E)\right\|_{l}\left\|u^{N}(E)\right\|_{l} \geq c l .
$$

Proof. Since the Wronskian is constant, it follows that

$$
W\left[u^{D},\left(u^{N}\right)^{*}\right](n)=c\left(u_{1, n+1}^{D} u_{2, n}^{N}-u_{1, n+1}^{N} u_{2, n}^{D}\right)=W\left[u^{D},\left(u^{N}\right)^{*}\right](-1)=c
$$

for every $n \geq 0$. Thus,

$$
\begin{aligned}
\sum_{n=0}^{l-1} c & =\sum_{n=0}^{l-1} W\left[u^{D},\left(u^{N}\right)^{*}\right](n) \leq\left|\left\langle\left(\begin{array}{c}
\left(u_{1, n+1}^{D}(E)\right)^{*} \\
\left(u_{2, n}^{D}(E)\right)^{*}
\end{array}\right),\left(\begin{array}{c}
u_{2, n}^{N}(E) \\
-u_{1, n+1}^{N}(E)
\end{array}\right)\right\rangle_{l-1}\right| \\
& \leq\left\|\left(\begin{array}{c}
u_{1, n+1}^{D}(E) \\
u_{2, n}^{D}(E)
\end{array}\right)\right\|_{l-1}\left\|\left(\begin{array}{c}
u_{1, n+1}^{N}(E) \\
u_{2, n}^{N}(E)
\end{array}\right)\right\|_{l-1},
\end{aligned}
$$


where we have used Cauchy-Schwarz inequality in the last step. Hence,

$$
\left\|u^{D}(E)\right\|_{l}\left\|u^{N}(E)\right\|_{l} \geq c l
$$

which concludes the proof of the lemma.

By taking into account the above adaptations to the Dirac operator, the proofs of Corollaries 4.7(a) and 4.7(b) follow the same lines of the proofs of Corollaries 4.4 and 4.5 in Ref. 12, respectively.

Corollary 4.7: (a) Suppose that for some $\alpha \in[0,1)$ and every $E$ in some Borel set $F$, every solution $\Psi$ to the Dirac eigenvalue equation (3.1) obeys

$$
\limsup _{l \rightarrow \infty} \frac{\|\Psi\|_{l}^{2}}{l^{2-\alpha}}<\infty
$$

Then, the restriction $\rho(F \cap \cdot)$ is $\alpha$-continuous.

(b) Suppose that

$$
\liminf _{l \rightarrow \infty} \frac{\left\|u^{D}(E)\right\|_{l}^{2}}{l^{\alpha}}=0
$$

is satisfied for every $E$ in some Borel set $F$. Then the restriction $\rho(F \cap \cdot)$ is $\alpha$-singular.

Corollary 4.7 can be rewritten in terms of the one-dimensional $2 \times 2$ transfer matrices $T(n ; E)$ defined by (3.3). This approach, based on Corollary 3.7 in Ref. 1, is of particular importance in our problem, since we have obtained in Sec. III the exact behavior of these matrices for a sparse potential like (1.5).

Corollary 4.8: Suppose that for some $\alpha \in[0,1)$ and every $E$ in some Borel set $A \subset \mathbb{R}$,

$$
\limsup _{l \rightarrow \infty} \frac{1}{l^{2-\alpha}} \sum_{n=0}^{l}\|T(n ; E)\|^{2}<\infty,
$$

with $\|\cdot\|$ some matrix norm. Then the restriction $\rho(A \cap \cdot)$ is $\alpha$-continuous.

Proof: By choosing $\theta_{1}=0$ and $\theta_{2}=\pi / 2$, it follows from a straightforward adaptation of Theorem 2.3 in Ref. 14 that there exists a constant $D$ such that

$$
\|T(n-1 ; E)\| \geq C \max \left\{R_{n}(0), R_{n}(\pi / 2)\right\},
$$

where $R_{n}(\theta)$ is the Prüfer radius at $n$ starting from the initial condition $\mathbf{v}_{\theta}=\left(\begin{array}{c}\cos \theta \\ \sin \theta\end{array}\right)$; explicitly, $C=\sqrt{\frac{\left(E+m c^{2}\right)\left(2 c+E-m c^{2}\right)}{\left(E-m c^{2}\right)\left(2 c-E-m c^{2}\right)}}$. Since

$$
\begin{aligned}
R_{n}^{2}\left(\theta_{1(2)}\right) & =\frac{4 c^{2}\left(E-m c^{2}\right)}{\left(E+m c^{2}\right)\left(4 c^{2}+m^{2} c^{4}-E^{2}\right)}\left|u_{1, n}^{D(N)}\right|^{2}+\frac{4 c^{2}}{\left(4 c^{2}+m^{2} c^{4}-E^{2}\right)}\left|u_{2, n-1}^{D(N)}\right|^{2} \\
& -\frac{4 c\left(E-m c^{2}\right)}{4 c^{2}+m^{2} c^{4}-E^{2}} \Re\left\{u_{1, n}^{D(N)}\left(u_{2, n-1}^{D(N)}\right)^{*}\right\}
\end{aligned}
$$

(see (3.7)), we obtain the inequality

$$
D\left[\left|u_{1, n}^{D(N)}\right|^{2}+\left|u_{2, n-1}^{D(N)}\right|^{2}\right] \leq R_{n}^{2}\left(\theta_{1(2)}\right),
$$


with $D=\frac{2 c\left(E-m c^{2}\right)\left(2 c-E-m c^{2}\right)}{\left(E+m c^{2}\right)\left(4 c^{2}+m^{2} c^{4}-E^{2}\right)}$. Thus,

$$
\sum_{n=0}^{l}\|T(n ; E)\|^{2} \geq C_{1} \max \left\{\left\|u^{D}(E)\right\|_{l+1}^{2},\left\|u^{N}(E)\right\|_{l+1}^{2}\right\},
$$

$C_{1}=C \times D$. Hypothesis (4.8), together with (4.9), imply Corollary 4.8.

Note that the growth of the norm of the transfer matrix gives exactly the growth of the increasing solution. This fact will be of great importance later on.

\section{B. Hausdorff dimension and spectral transition}

The last step in the determination of the Hausdorff dimension of the measure $\rho$ is the following extension of Proposition 3.9 in Ref. 1:

Proposition 4.9: Let $\mathcal{A}=\left(a_{n}\right)_{n \geq 1}$ be given by (1.7), $E \in \mathbb{R}$ and assume that the sequence $\left(\theta_{n}^{\omega}\right)_{n \geq 1}$ of Prüfer angles (3.12) is u.d. $\bmod \pi$ for every $\theta_{0} \in[0, \pi)$, almost every $\varphi \in[0, \pi)$ (with respect to Lebesgue measure) and almost every $\omega \in \Xi$. Then, there is a generalized eigenfunction $\Psi$ (i.e., $\Psi$ satisfies $H_{v, \phi}(m, c) \Psi=E \Psi$ and the phase boundary condition (1.4)) such that

$$
C_{n}^{-1} r^{n / 2} \leq R_{n}(\phi) \leq C_{n} r^{n / 2}
$$

holds with $r$ given by (3.16) and $C_{n}^{1 / n} \searrow R_{0}(\phi)$ as $n \rightarrow \infty$. In addition, there exists a subordinate solution $\Phi$ (with $\alpha^{*}$-phase boundary condition) for energy $E$ such that, for all sufficiently large $n$, the Prüfer radius associated with $\Phi$ satisfies

$$
\left|R_{n}\left(\alpha^{*}\right)\right| \leq \tilde{C}_{n} r_{j}^{-n / 2}
$$

with $\tilde{C}_{n}^{1 / n} \searrow R_{0}\left(\alpha^{*}\right)$ as $n \rightarrow \infty$.

Proof: The proof of the proposition follows the same steps of the proof of Proposition 3.9 in Ref. 1. We, nevertheless, trace them out.

The first part of the proposition is simply Lemma 3.4. The second part follows the same steps of Lemma 3.6 in Ref. 2, which adapts the results presented in Theorem 8.1 in Ref. 19 (the latter gives a sufficient condition for the existence of a subordinate solution to the Dirac equation (3.1)).

The inequalities (4.10) and the results of Theorem 2.3 in Ref. 14 imply that

$$
C_{n}^{-1} r^{n / 2} \leq t_{n} \leq C_{n} r^{n / 2}
$$

with $t_{n}:=\left\|T\left(a_{n}+1 ; E\right)\right\|$ and $r$ given by (3.16). From now on, the proof of the existence of a subordinate solution, as well as the determination of its asymptotic behavior, follow from the proof of Lemma 3.6 in Ref. 2.

Remark 4.10: The corresponding modifications of the known results for Schrödinger operators, employed in the proof of Proposition 4.9 to the Dirac setting studied in this work, are straightforward, being therefore omitted.

Finally, we are able to prove our main result.

Proof: (Theorem 1.4) We base the arguments on the proof of Theorem 3.11 in Ref. 1. First, introduce the notation

$$
A=-\sqrt{m^{2} c^{4}+2 c^{2}(1-\cos (\mathbb{Q} \pi))} \cup \sqrt{m^{2} c^{4}+2 c^{2}(1-\cos (\mathbb{Q} \pi))},
$$

which will also be used in other occasions ahead.

Theorem 3.6 implies that the sequence $\left(\theta_{n}^{\omega}\right)_{n \geq 0}$ of Prüfer angles is u.d.mod $\pi$ for every $\theta_{0} \in[0, \pi]$, every $E \in \mathrm{L}^{\prime} \equiv L \backslash A$, and almost every $\omega \in \Xi$. We obtain from (4.12) the estimates

$$
\|T(k ; E)\| \leq C_{n} r^{n / 2} \leq C_{n}^{\prime} a_{n}^{\gamma / 2} \leq C_{n}^{\prime \prime} k^{\gamma / 2},
$$

which hold for every $E \in \mathrm{L}^{\prime}$ and every $a_{n}^{\omega} \leq k<a_{n+1}^{\omega}$, with $\gamma \equiv \ln r / \ln \beta, C_{n}^{\prime \prime}>0$ and $\lim _{n \rightarrow \infty}\left(C_{n}^{\prime \prime}\right)^{1 / n}<\infty$. 
It follows by the constancy of $\|T(k ; E)\|$ on $\left[a_{n}+1, a_{n+1}\right]$ (since the free matrix $T_{0}$ is equivalent to a rotation; see Sect. III) that

$$
\sum_{k=0}^{l}\|T(k ; E)\|^{2} \leq c l^{1+\gamma}
$$

holds for some $c>0$ and every $E \in \mathrm{L}^{\prime}$.

The application of Proposition 4.9 together with (1.10) guarantees, for these values of $E$, the existence of a subordinate solution $\Phi^{\text {sub }}$ such that its sequence of Prüfer radii satisfies the estimate

$$
\left|R_{n}\left(\theta_{\alpha^{*}}\right)\right| \leq C_{n}^{\prime \prime \prime} a_{n}^{-\gamma / 2},
$$

for some constant $C_{n}^{\prime \prime \prime}>0$.

Since every solution to (3.1) has constant modulus on the interval $\left[a_{n}+1, a_{n+1}\right]$, we have

$$
\left\|\Phi^{\text {sub }}\right\|_{l}^{2} \leq c^{\prime} l^{1-\gamma},
$$

for some $c^{\prime}>0$.

Since the restriction of the measure $\rho$ to $I$ is supported on the set of those $E$ for which $\Phi^{\text {sub }}$ satisfies the boundary condition $\phi$ (thanks to the fact that $\rho$ has no absolutely continuous part; see Theorem 1 in Ref. 11), we have $u^{D}=\Phi^{\text {sub }}$ for almost every $E \in I$ with respect to $\rho$ and for almost every boundary condition $\phi$ (by the theory of rank-one perturbation; see Theorem 1.3 in Ref. 12).

Thus, by (4.14) and (4.15), on the intervals (2.13), one has

$$
\limsup _{l \rightarrow \infty} \frac{1}{l^{2-\alpha}} \sum_{k=0}^{l}\|T(k ; E)\|^{2}<\infty
$$

and

$$
\liminf _{l \rightarrow \infty} \frac{\left\|u^{D}(E)\right\|_{l}^{2}}{l^{\alpha^{\prime}}}=0,
$$

provided $2-\alpha=1+\gamma+\varepsilon$ and $\alpha^{\prime}=1-\gamma+\varepsilon$, respectively, where $\varepsilon$ is an arbitrary positive number.

It follows, by Corollary 4.8, that the spectral measure $\rho$ is simultaneously $(1-\gamma-\varepsilon)$ continuous and $(1-\gamma+\varepsilon)$-singular. Since $\varepsilon$ is arbitrary, we have from Remark 4.2 that the restriction $\rho\left(I^{\prime} \cap \cdot\right)$ has exact Hausdorff dimension given by (1.11), where $I^{\prime} \subseteq \mathrm{L}^{\prime}$.

Finally, from the theory of rank one perturbations (more specifically, Theorem 8.1 of $^{26}$ ), we know that $\rho(A)=0$ (see (4.13)) holds for almost every $\phi$; therefore, for almost every $\phi$, the restriction $\rho(I \cap \cdot)$ has (1.11) as its Hausdorff dimension. This concludes the proof of the theorem.

Remark 4.11: We have checked that it is possible to extend the results of Refs. 11 and 13 to the Dirac setting, in the same way as exposed here for the results of generalized subordinacy discussed in Ref. 12.

Despite the obtained exact Hausdorff dimension of the spectral measure $\rho$, what can we infer from the spectral nature of the operator $H_{v, \phi}(m, c)$ on the interval (2.13)? This is a tricky question, since we are induced to think that for $\alpha_{\rho}=0$, the spectrum is simply dense pure point and for $\alpha_{\rho}>0$, the spectrum is singular continuous. There are, however, some examples in the literature $2,9,31$ where the spectrum is singular continuous with null Hausdorff dimension. Note that Theorem 1.5 settles the problem. Now we present its proof.

Proof: (Theorem 1.5) We must prove, for fixed $v$ and $\beta$, the inclusion

$$
I_{1} \backslash A_{1} \subseteq\left\{E \in \mathbb{R}: \sum_{n=0}^{\infty}\|T(n ; E)\|^{-2}=\infty\right\}
$$


( $A_{1}$ is a set with zero Lebesgue measure), since (a) follows directly from an adaptation of Theorem 2.1 in Ref. 27. Now, if

$$
\left[I_{1} \backslash A_{1}\right]^{C} \subseteq\left\{E \in \mathbb{R}: \sum_{n=1}^{\infty} \beta^{n}\left(t_{n}\right)^{2}\left(\sum_{m=n}^{\infty}\left(t_{m}^{j}\right)^{-2}\right)^{2}<\infty\right\},
$$

then (b) follows from an adaptation of Lemma 4.1 in Ref. 2 and by Proposition 4.2 in Ref. 20.

We pick $n$ such that $a_{N}^{\omega} \leq n<a_{N+1}^{\omega}$ is valid for some $N \in \mathbb{N}$. We obtain from (1.7) and (3.4),

$$
\sum_{m \leq n}\|T(m ; E)\|_{U}^{-2}=\sum_{k \leq N+1}\left\|T\left(a_{k}^{\omega} ; E\right)\right\|_{U}^{-2} \beta^{k+1}+\left\|T\left(a_{N}^{\omega} ; E\right)\right\|_{U}^{-2} \sum_{a_{N+1}^{\omega} \leq m<n} 1,
$$

with $\|\cdot\|_{U}:=\left\|U \cdot U^{-1}\right\|$ (see Sec. 4 of Ref. 20).

We have by Theorem 2.3 in Ref. 14 the inequalities

$$
\|T(m ; E)\|^{-2} \leq C^{2}\left\|T\left(a_{k}^{\omega} ; E\right)\right\|_{U}^{-2} \leq C^{2}\left(\max _{i \in\{1,2\}} R_{k}\left(\theta_{i}\right)\right)^{-2}
$$

and

$$
\|T(m ; E)\|^{-2} \geq C^{-2}\left\|T\left(a_{k}^{\omega} ; E\right)\right\|_{U}^{-2} \geq \tilde{C}^{-2}\left(\max _{i \in\{1,2\}} R_{k}\left(\theta_{i}\right)\right)^{-2},
$$

for every $a_{k}^{\omega} \leq m<a_{k+1}^{\omega}$, with $C$ as in the proof of Corollary 4.8 and

$$
\tilde{C}=C /\left|\sin \left(\theta_{1}-\theta_{2}\right) / 2\right|
$$

$\left(\theta_{1}, \theta_{2} \in[0, \pi)\right.$ represent a pair of initial Prüfer angles such that $0<\left|\theta_{1}-\theta_{2}\right|<\pi / 2$; see Lemma 2.2 of Ref. 14).

Introduce

$$
S_{N, M}^{ \pm}(\beta, \varphi) \equiv \sum_{k=N}^{M} C_{k}^{ \pm}\left(\beta^{k+1} \pm 2 k\right) r^{-k}
$$

(the term $2 k$ is due to the uncertainty associated with the position of the $k$ th barrier: $\beta^{k+1}-2 k$ $\leq a_{k}^{\omega} \leq \beta^{k+1}+2 k-1$ for all $\omega \in \Xi$ ), with $r$ given by (3.16). It follows by Lemma 3.4, and Eqs. (4.16), (4.18), and (4.19),

$$
\tilde{C}^{-2} S_{N+1, M}^{-} \leq \sum_{m=n}^{a_{M}+1}\|T(m ; E)\|^{-2} \leq C^{2} S_{N, M}^{+},
$$

for every $M \geq N+1$ and $E \in B$, with

$$
B \equiv-\sqrt{m^{2} c^{4}+2 c^{2}(1-\cos ([0, \pi] \backslash \mathbb{Q} \pi))} \cup \sqrt{m^{2} c^{4}+2 c^{2}(1-\cos ([0, \pi] \backslash \mathbb{Q} \pi))},
$$

$2 \cos (\mathbb{Q} \pi \cap[0, \pi))$ representing the "energies" where the sequence $\left(\theta_{n}^{\omega}\right)_{n \geq 0}$ is not u. d. $\bmod \pi$; see the proof of Theorem 3.6.

It is clear by Lemma 3.4, and Eqs. (3.16), (4.20), and (4.21) that

$$
B \subseteq\left\{E: \lim _{n \rightarrow \infty}\|T(n ; E)\|=\infty\right\},
$$

i.e., $B$ belongs to the complement of the essential support of the absolutely continuous part of the spectrum of $H_{v, \phi}(m, c), \Sigma_{\mathrm{ac}}$, according to Theorem 1.1 of Ref. 19. Hence, except for the set $A_{1}=(\mathbb{Q} \pi \cap[0, \pi)) \cup A^{*}$ of Lebesgue zero measure $\left(A^{*}\right.$ is some set of Lebesgue zero measure presented in the definition of $\Sigma_{\mathrm{ac}}$ ), the set

$$
B_{1} \equiv-\sqrt{m^{2} c^{4}+2 c^{2}\left(1-\cos \left([0, \pi] \backslash A_{1}\right)\right)} \cup \sqrt{m^{2} c^{4}+2 c^{2}\left(1-\cos \left([0, \pi] \backslash A_{1}\right)\right)}
$$


belongs to the singular spectrum. It follows from (4.19) and the left-hand member of (4.20), for $M \rightarrow \infty$, that

$$
\sum_{n=0}^{\infty}\|T(n ; E)\|^{-2}=\infty
$$

if $\beta>r$. Therefore, we conclude from (4.22) and Theorem 2.2 of Ref. 14 that the essential spectrum of $H_{v, \phi}(m, c)$ is purely singular continuous when restricted to $I \backslash A_{1}$, and this proves part (a) of the theorem.

We prove part (b) by assuming that $\beta<r$. By introducing

$$
S=\sum_{m=n} C_{m} r^{-m}
$$

it follows by Lemma 3.4 and Eqs. (4.16) and (4.17), that

$$
\sum_{m=n}^{\infty}\left\|T\left(a_{m}+1 ; E\right)\right\|^{-2} \leq C^{2} S .
$$

Finally, we have from Lemma 3.4 and Eqs. (4.17), (4.18), (4.23), and (4.24) that

$$
\begin{aligned}
\sum_{n=1}^{\infty}\left(\beta^{n}+\omega_{n}-\omega_{n-1}\right)\left\|T\left(a_{n}+1 ; E\right)\right\|^{2} & \left(\sum_{m=n}^{\infty}\left\|T\left(a_{m}+1 ; E\right)\right\|^{-2}\right)^{2} \\
& \leq C^{\prime} \sum_{n=1}^{\infty}\left(\beta^{n}+2 n\right) C_{n} r^{n} S^{2} \\
\leq & C^{\prime \prime} \sum_{n=1}^{\infty}\left(\beta^{n}+2 n\right)\left(C_{n}\right)^{3} r^{-n}
\end{aligned}
$$

converges for every $E \in I^{C}$. It follows by Lemma 3.6 in Ref. 2 and (4.25) above, that if $E \in I^{c} \backslash A_{1}$, then the Dirac equation (3.1) have a $l^{2}\left(\mathbb{Z}_{+}, \mathbb{C}^{2}\right)$ solution. Since, by Theorem 1.3, $I^{c} \in \sigma_{\text {ess }}\left(H_{v, \phi}(m, c)\right)$ for every $\phi \in[0, \pi]$, the hypothesis of Proposition 4.2 of Ref. 20 is fulfilled and consequently $H_{v, \phi}(m, c)$ have only dense pure point spectrum in $I^{C}$ for almost every $\phi \in[0, \pi]$. This concludes the proof of part (b) of the theorem.

\section{ACKNOWLEDGMENTS}

The work of S. L. Carvalho is supported by FAPESP under Grant No. 2010/10236-3. The work of C. R. de Oliveira is partially supported by CNPq. The work of R. A. Prado is partially supported by PROPe/UNESP and FUNDUNESP.

${ }^{1}$ Carvalho, S. L., Marchetti, D. H. U., and Wreszinski, W. F., "Sparse Block-Jacobi matrices with accurate Hausdorff dimension," J. Math. Anal. Appl. 368, 218 (2010).

${ }^{2}$ Carvalho, S. L., Marchetti, D. H. U., and Wreszinski, W. F., "On the uniform distribution of the Prüfer angles and its implication to a sharp transition of Jacobi matrices with randomly sparse perturbations," e-print arXiv:1006.2849.

${ }^{3}$ Coddington, E. A. and Levinson, N., Theory of Ordinary Differential Equations (Krieger Publishing Company, Malabar, 1984) (reprint edition).

${ }^{4}$ Combes, J. M. and Mantica, G., "Fractal dimensions and quantum evolution associated with sparse potential Jacobi matrices," in Long time behaviour of classical and quantum systems, Series on Concrete and Applied Mathematics Vol. 1, edited by S. Graffi and A. Martinez (World Scientific, Singapore, 2001), pp. 107-123.

${ }^{5}$ Cycon, H. L., Froese, R. G., Kirsch, W., and Simon, B., Schrödinger Operators with Application to Quantum Mechanics and Global Geometry (Springer, Berlin, 1987).

${ }^{6}$ de Oliveira, C. R., Intermediate Spectral Theory and Quantum Dynamics (Birkhäuser, Basel, 2009).

${ }^{7}$ de Oliveira, C. R. and Prado, R. A., "Dynamical localization for the 1D Bernoulli discrete Dirac operator," J. Phys. A 38, L115 (2005).

${ }^{8}$ de Oliveira, C. R. and Prado, R. A., "Spectral and localization properties for the one dimensional Bernoulli discrete Dirac operator,” J. Math. Phys. 46, 072105 (2005). 
${ }^{9}$ Del Rio, R., Jitomirskaya, S., Last, Y., and Simon, B., "Operators with singular continuous spectrum, IV. Hausdorff dimension, rank one perturbations and localization,” J. Anal. Math. 69, 153 (1996).

${ }^{10}$ Falconer, K. J., Fractal Geometry (Wiley, Chichester, 1990).

${ }^{11}$ Gilbert, D. J. and Pearson, D. B., "On subordinacy and analysis of the spectrum of one-dimensional Schrödinger operators," J. Math. Anal. Appl. 128, 30 (1987).

${ }^{12}$ Jitomirskaya, S. and Last, Y., "Power-law subordinacy and singular spectra I. Half-line operators," Acta. Math. 183, 171 (1999).

${ }^{13}$ Khan, S. and Pearson, D. B., "Subordinacy and spectral theory for infinite matrices," Helv. Phys. Acta 65, 505 (1992).

${ }^{14}$ Kiselev, A., Last, Y., and Simon, B., "Modified Prüfer and EFGP transforms and the spectral analysis of one-dimensional Schrödinger operators," Commun. Math. Phys. 194, 1 (1998).

${ }^{15}$ Kuipers, L. and Niederreiter, H., Uniform Distributions of Sequences (Wiley, New York, 1974).

${ }^{16}$ Krutikov, D., "Asymptotics of the Fourier transform of the spectral measure for Schrödinger operators with bounded and unbounded sparse potentials," J. Phys. A 35, 6393 (2002).

${ }^{17}$ Last, Y., "Quantum dynamics and decomposition of singular continuous spectra," J. Funct. Anal. 142, 406 (1996).

${ }^{18}$ Last, Y., "Sturm-Liouville operator on infinite intervals," in Sturm-Liouville Theory: Past and Present, edited by W. O. Amrein, A. M. Hinz, and D. B. Pearson (Birkhäuser Verlag, Basel, 2005), pp. 99-120.

${ }^{19}$ Last, Y. and Simon, B., "Eigenfunctions, transfer matrices, and absolutely continuous spectrum of one-dimensional Schrödinger operators," Invent. Math. 135, 329 (1999).

${ }^{20}$ Marchetti, D. H. U., Wreszinski, W. F., Guidi, L. F., and Angelo, R. M., "Spectral transition in a sparse model and a class of nonlinear dynamical systems," Nonlinearity 20, 765 (2007).

${ }^{21}$ Pearson, D. B., "Singular continuous measures in the scattering theory," Commun. Math. Phys. 60, 13 (1978).

${ }^{22}$ Prado, R. A. and de Oliveira, C. R., "Sparse 1D discrete Dirac operators I: Quantum transport," (preprint), J. Math. Anal. Appl. (submitted).

${ }^{23}$ Rogers, C. A. and Taylor, S. J., "The analysis of additive set functions in Euclidean space,” Acta. Math. 101, 273 (1959).

${ }^{24}$ Saks, S., Theory of the Integral, 2nd ed. (Hafner, New York, 1937).

${ }^{25}$ Sakurai, J. J., Advanced Quantum Mechanics (Addison-Wesley, New York, 1967).

${ }^{26}$ Simon, B., Spectral Analysis of Rank One Perturbations and Applications, CRM Proceeding and Lecture Notes Vol. 8, edited by J. Feldman, R. Froese, andL. Rosen (American Mathematical Society, Providence, RI, 1995), pp. 109-149.

${ }^{27}$ Simon, B. and Stolz, S., "Operators with singular continuous spectrum. V. Sparse potentials," Proc. Am. Math. Soc. 124, 2073 (1996).

${ }^{28}$ Tcheremchantsev, S., "Dynamical analysis of Schrödinger operators with growing sparse potentials," Commun. Math. Phys. 253, 221 (2005).

${ }^{29}$ Thaller, B., The Dirac Equation (Springer-Verlag, Heidelberg, 1992).

${ }^{30}$ Teschl, H., Jacobi Operators and Completely Integrable Nonlinear Lattices (American Mathematical Society, New York, 2000).

${ }^{31}$ Zlatloš, A., "Sparse potentials with fractional Hausdorff dimension,” J. Funct. Anal. 207, 216 (2004). 